In the contrary case, let the original diagram oklmn be arbitrary. Applying a transformation of the aforesaid kind which changes $m, n$ into $m_{1}, n_{1}$, let us consider the two intermediate triangles olm, onk, which are changed into $o l m_{1}, o n_{1} k$. On the basis $l m_{1}$, we can construct a triangle $l m_{1} o_{1}$ directly similar to $l m o$ and, on the basis $n_{1} k$, the triangle $n_{1} k o_{1}^{\prime}$ directly similar to $n k o$; in general, the two new vertices $o_{1}, o_{1}^{\prime}$ will be different. They will coincide if the original diagram is a reduced one. ${ }^{9}$

New York Crty

9 The problem would require further investigation because there is no reason not to consider the complete quadrilateral formed by the sides of $q$ and, therefore, we ought to apply the present considerations to three diagonals instead of two.

\title{
NOTE ON CONVEX SPHERICAL CURVES
}

\section{A. SANTALó}

1. Introduction. The formula

$$
L=\int_{0}^{\pi} \alpha d \tau
$$

for plane convex curves in which $L$ is the length and $\alpha$ the breadth according to the direction $\tau$ is well known [2, p. 65].1

The principal object of the present note is to obtain the formula (8) which generalizes (1) to convex curves on the sphere of unit radius and to deduce from this some consequences.

2. Principal formula. Let us consider the sphere of unit radius. A closed curve on the sphere is said to be convex when it cannot be cut by a great circle in more than two points. It is well known that a convex curve divides the surface of the sphere into two parts, one of which is always wholly contained in a hemisphere; that is, there is always a great circle which has the whole convex curve on the same side. When we say the area of a convex curve $K$ we understand the area of that part of the surface of the sphere which is bounded by $K$ and is smaller than or equal to a hemisphere.

Let $K$ be a convex curve on the sphere of unit radius of length $L$ and area $F(L \leqq 2 \pi, F \leqq 2 \pi)$. The great circles which have only one common point or include a complete segment common with the curve

Received by the editors January 21, 1944.

1 Numbers in brackets refer to the references cited at the end of the paper. 
$K$ are called "great circles of support" of $K$. In each point of $K$ for which there exists a tangent great circle the great circle of support coincides with this.

Let $\tau$ be the angle between a fixed and a variable great circle of support and $s$ the arc of $K$. If $\rho_{g}$ is the radius of geodesic curvature of $K$ the following formulas are known [4, p. 712],

$$
\rho_{g}=d s / d \tau, \quad \int_{L} d s / \rho_{g}=\int_{K} d \tau=2 \pi-F .
$$

Let $C$ be a great circle which cuts $K$. We suppose $C$ "oriented" and to each orientation we make correspond one of the points $P_{c}$ of the two in which the diameter perpendicular to $C$ cuts the sphere. If we consider all the great circles which cut $K$ (each one of these counted twice to correspond with the two orientations) the area covered by the points $P_{c}$ has the value $2 L[4, \mathrm{p} .709]$; that is to say, if $d P_{c}$ represents the element of area of the sphere of unit radius corresponding to the point $P_{c}$,

$$
\int_{C \cdot K \neq 0} d P_{c}=2 L .
$$

The convex curve $K$ being fixed, the oriented great circle $C$ or, what is the same, the point $P_{c}$, can be determined by the point $A$ of $K$ in which the great circle orthogonal to a circle of support of $K$ is also orthogonal to $C$, and by the distance $a$ from $A$ to $C$ (Fig. 1). If $\tau$ is

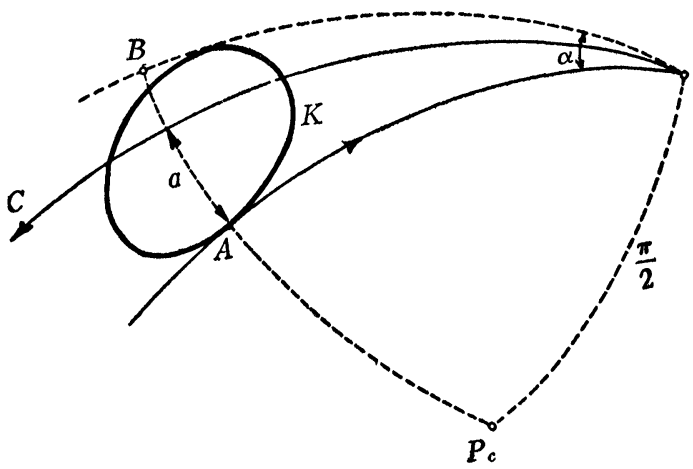

FIG. 1

the angle which determines the great circle of support of $K$ in the point $A$, the coordinates to determine $P_{c}$ will be $a, \tau$. We must then express the element of area $P_{c}$ as a function of the coordinates $a, \tau$. 
Suppose first that $a$ remains fixed and $\tau$ passes to $\tau+d \tau$. The point $P_{c}$ will describe an arc $P_{c} P_{c}^{\prime}=d \sigma$, the value of which is (Fig. 2)

$$
d \sigma=\sin (\pi / 2-a+\phi) d \psi=\cos (a-\phi) d \psi,
$$

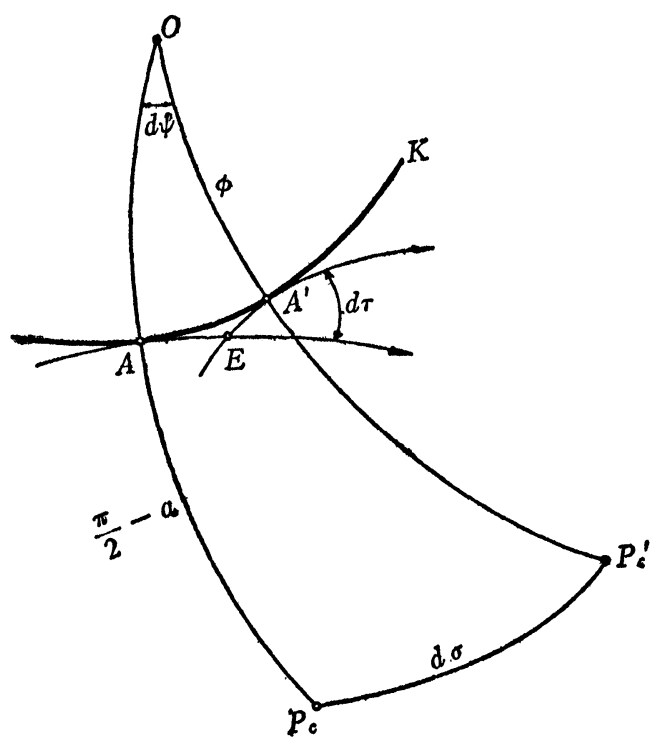

FIG. 2

$\phi=A O$ being the radius of spherical curvature of $K$ at the point $A$. As a function of the radius of geodesic curvature it is

$$
\tan \phi=\rho_{o}=d s / d \tau .
$$

The element $d \psi=A O A^{\prime}$ is the angle between the great circles orthogonal to $K$ in $A$ and $A^{\prime}$. The area of the quadrilateral $A O A^{\prime} E$ has the value $(\pi-d \tau)+d \psi+\pi-2 \pi=d \psi-d \tau$. But save for infinitesimals of secondary order the same area is also equal to the area of the circular sector $A O A^{\prime}$ which has the value $(1-\cos \phi) d \psi$. In consequence

$$
d \tau=\cos \phi d \psi .
$$

If $\tau$ remains fixed and $a$ passes to $a+d a$, the point $P_{c}$ describes an arc $d a$ upon the great circle $P_{c} A$. Therefore, taking into account (4) and (6) the element of area $d P_{c}$ will be expressed

$$
d P_{\mathrm{c}}=d \sigma d a=(\cos (a-\phi) / \cos \phi) d a d \tau,
$$


that is to say, in accordance with (5),

$$
d P_{c}=\cos a d a d \tau+\sin a d a d s .
$$

Let us substitute this expression in (3). For each value of $s$ (or $\tau$ ) the arc $a$ can vary from 0 to the breadth $\alpha$ of $K$ corresponding to the point $s$ (or direction $\tau$ ). Therefore

$$
\int_{C \cdot K \neq 0} d P_{c}=\int_{K} d \tau \int_{0}^{\alpha} \cos a d a+\int_{L} d s \int_{0}^{\alpha} \sin a d a,
$$

or, in accordance with (3),

$$
L=\int_{K} \sin \alpha d \tau-\int_{L} \cos \alpha d s .
$$

This is the principal formula we wished to obtain; it is valid for any convex curve $K$ on the sphere of unit radius. The breadth $\alpha$ corresponding to a point $A$ of $K$ is equal to the $\operatorname{arc} A B$ (Fig. 1) of the great circle orthogonal to $K$ at the point $A$ comprehended between $A$ and the next point of intersection with another great circle of support of $K$ also orthogonal to $A B$.

3. Dual formula. By duality $[4$, p. 710$]$ to the breadth $\alpha$ corresponds the arc $\pi-h, h$ being the arc $A E$ (Fig. 3 ) of the great circle

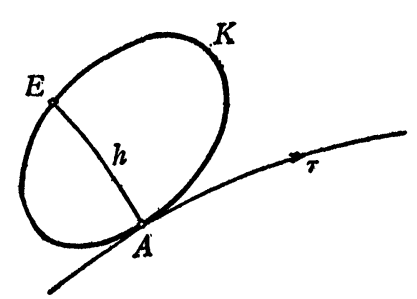

FIG. 3

orthogonal to $K$ at the point $A$ which is interior to $K$. Moreover the length $L$ must be replaced by $2 \pi-F, d s$ by $d \tau$ and $d \tau$ by $d s$. Therefore transforming by duality the formula (8) we shall obtain

$$
2 \pi-F=\int_{L} \sin h d s+\int_{K} \cos h d \tau .
$$

4. Consequences. (a) Let $\beta$ be the minimum breadth of $K$, that is to say the minimum value of $\alpha$, and $\delta$ the maximum value of $\alpha ; \delta$ is equal to the "diameter" of $K$. Let us suppose that $\beta \leqq \alpha \leqq \delta \leqq \pi / 2$. Then $\sin \delta \geqq \sin \alpha, \cos \delta \leqq \cos \alpha$, and from (8) and (2) we deduce 


$$
L \leqq(2 \pi-F) \sin \delta-L \cos \delta
$$

from which we have

$$
L /(2 \pi-F) \leqq \tan (\delta / 2) .
$$

Similarly, $\sin \alpha \geqq \sin \beta, \cos \alpha \leqq \cos \beta$, and consequently

$$
L /(2 \pi-F) \geqq \tan (\beta / 2) .
$$

Therefore: On the sphere of unit radius for any convex curve $K$ of minimum breadth $\beta$ and diameter $\delta \leqq \pi / 2$, the inequalities (10) and (11) are verified.

(b). In (10) and (11) there is equality only when $\alpha=\beta=\delta=$ const., that is to say for the cases of convex curves of constant breadth. These curves of constant breadth on the sphere have been studied by Blaschke [1]. For such curves it is deduced from (8) that

$$
L=(2 \pi-F) \tan (\alpha / 2) \text {. }
$$

This formula, obtained also by Blaschke [1], appears here as a particular case of our general formula (8).

(c). It is well known that isoperimetric inequality $L^{2}+(2 \pi-F)^{2}$ $\geqq 4 \pi^{2}$ holds for any convex curve $K$ on the unit sphere and that the equality is only true for the circles $[4$, p. 718]. This isoperimetric inequality and (10), (11) give us

$$
F \leqq 2 \pi(1-\cos (\delta / 2)), \quad L \geqq 2 \pi \sin (\beta / 2) .
$$

Consequently: upon the sphere, given the diameter $\delta \leqq \pi / 2$ the convex curve of maximum area is the circle of radius $\delta / 2$; given the minimum breadth $\beta$ the curve of minimum length is the circle of radius $\beta / 2$.

(d). If the convex curve $K$ has continuous geodesic curvature, from (9) and (2) we deduce

$$
\int_{L} \sin (h / 2)\left(\rho_{o}^{-1} \sin (h / 2)-\cos (h / 2)\right) d s=0
$$

and we therefore have the theorem: In any convex curve on the sphere of unit radius with continuous radius of geodesic curvature there are at least two points for which

$$
\rho_{o}=\tan (h / 2),
$$

$h$ being the chord of $K$ orthogonal to $K$ at the point considered.

Analogously from (8) and (2) we deduce

$$
\int_{L} \cos (\alpha / 2)\left(\rho_{g}^{-1} \sin (\alpha / 2)-\cos (\alpha / 2)\right) d s=0
$$


and therefore: in any convex curve on the sphere of unit radius with continuous radius of geodesic curvature there are at least two points for which

$$
\rho_{g}=\tan (\alpha / 2),
$$

$\alpha$ being the breadth of $K$ corresponding to the point considered.

5. Passage to the case of the plane. Considering a sphere of radius $R$ and making $R \rightarrow \infty$, the anterior formulas transform themselves into valid formulas for plane convex curves.

The principal formula (8) if $K$ belongs to a sphere of radius $R$ is written

$$
\frac{L}{R}=\int_{K} \sin \frac{\alpha}{R} d \tau-\int_{L} \cos \frac{\alpha}{R} \frac{d s}{R} ;
$$

multiplying both sides by $R$ and making $R \rightarrow \infty$ we obtain the well known formula (1).

The formula (9) taking into account (2) may be written

$$
\int_{K} \sin ^{2} \frac{h}{2} d \tau=\int_{L} \sin \frac{h}{2} \cos \frac{h}{2} d s .
$$

Writing this formula for a sphere of radius $R$, multiplying afterwards both sides by $R^{2}$ and making $R \rightarrow \infty$ we obtain

$$
J=\int_{L} h d s=2^{-1} \int_{0}^{2 \pi} h^{2} d \tau .
$$

In this formula $h$ is the chord of the plane convex curve $K$ normal to this at the point $s$ (or $\tau$ ).

The element $h d s$ which appears in the first expression of $J$ in (16) is the area of a strip of $K$ with the height $h$ and base $d s$. If a point $P(x, y)$ interior to $K$ belongs to $\nu$ of these strips, from $P$ there will be $\nu$ normals to $K$ and the element of area $d x d y$ will be counted $\nu$ times in (16). Hence, if $\nu$ has a finite value for any $P$ interior to $K$, the integral $J$ is also equal to

$$
J=\iint_{F} \nu d x d y,
$$

$\nu$ being the number of normals to $K$ from the point $P(x, y)$ interior to $K$.

If there are points with $\nu=\infty$ (for instance the case of the circle) the integral (17) must be considered as the limit of the similar ones referring to convex curves $K_{n}$ (with $\nu$ always finite) which tend to $K$.

It is interesting to study the limits between which the integral $J$ 
is bounded. From any point $P(x, y)$ interior to $K$ may be traced always at least two normals to $K$, joining in straight lines $P$ with the points of $K$ of minimum and maximum distance to $P$. Consequently

$$
J \geqq 2 F \text {. }
$$

From another aspect, the second expression for $J$ in (16) shows that $J$ is equal to the area that is obtained by extending from a fixed point of the plane segments of length $h$ parallel to the direction $\tau$; as $h$ is less than or equal to the length of the maximum chord of $K$ which also has direction perpendicular to the line of support of direction $\tau$, a known theorem of Rademacher about vector regions of convex curves $[3 ; 2$, p. 105] tells us that

$$
J \leqq 6 F \text {. }
$$

(18) cannot be bettered, it being sufficient to consider a triangle with an angle approximating to $\pi$ in order that $J$ comes near $2 F$ in such degree as we should wish.

The inequality (19), on the contrary, is probably excessive. For the convex curves of constant breadth $\alpha$ we have

$$
J=L \alpha=\pi \alpha^{2} .
$$

If we recall that the triangle of Reuleaux [2, p. 132] is the figure which, given the constant breadth $\alpha$, has minimum area of value $\left(\pi-3^{1 / 2}\right) \alpha^{2} / 2$, we see that $F \geqq\left(\pi-3^{1 / 2}\right) \alpha^{2} / 2$ and consequently from (20) we deduce

$$
J \leqq 2 \pi F /\left(\pi-3^{1 / 2}\right)=4,4576 \cdots F .
$$

It is probable that this superior bound is valid not only for convex curves of constant breadth but also for any convex curve of the plane. Nevertheless I have not been able to prove this last assertion.

\section{REFERENCES}

1. Blaschke, W. Einige Bemerkungen iiber Kurven und Flächen konstanter Breite. Bericht taber die Verhandlungen der Sächsischen Akademie der Wissenschaften vol. 67 (1915).

2. Bonnesen, T., and Fenchel, W., Theorie der konvexen Körper, Ergebnisse der Mathematik und ihrer Grenzgebiete, Berlin, 1934.

3. Rademacher, H., Ueber den Vektorenbereich eines konvexen ebenen Bereiches, Jber. Deutschen Math. Verein. vol. 34 (1925).

4. Santal6, L. A., Integral formulas in Crofton's style on the sphere and some inequalities referring to spherical curves, Duke Math. J. vol. 9 (1942) pp. 707-722.

Universidad NaCional DEL Litoral, Rosario 